\title{
Congenital Cyanotic Cardiac Surgery in Children: Is Algorithm-Based Point-of-Care Testing Essential to Prevent Bleeding?
}

\author{
Vandana Bhardwaj ${ }^{1}$ Poonam Malhotra Kapoor ${ }^{1}$ \\ Milind Padmakar Hote ${ }^{2}$ Palleti Rajashekhar ${ }^{2}$ \\ ${ }^{1}$ Department of Cardiothoracic Vascular Anaesthesia, All India \\ Institute of Medical Sciences, New Delhi, India \\ 2Department of Cardiothoracic Vascular Surgery, All India Institute \\ of Medical Sciences, New Delhi, India \\ J Card Crit Care TSS 2018;2:84-90
}

Ameya Anand Karanjkar ${ }^{1} \quad$ Ujjwal Kumar Chowdhury ${ }^{2}$

Address for correspondence Vandana Bhardwaj, MD, Department of Cardiothoracic Vascular Anaesthesia, All India Institute of Medical Sciences, New Delhi 110029, India (e-mail: drvandana291@gmail.com).

\section{Abstract}

\section{Keywords}

- congenital cyanotic heart disease

- rotational thromboelastometry

- thrombocytopenia

- hypofibrinogenemia

- thromboelastography
Background Patients with cyanotic congenital heart disease (CCHD) have multifactorial hematologic abnormalities. In continuation of our previous study titled "Coagulopathies in Cyanotic Cardiac Patients: An Analysis with Three Point-of-Care Testing Devices (Thromboelastography, Rotational Thromboelastometry, and Sonoclot Analyzer)," we extended this prospective observational study to a larger cohort to reconfirmed the need to do a point-of-care (POC) test in bleeding cyanotic children. We formulated an algorithm. We conducted this study now by comparing three different POC parameters in an algorithm-based manner and deciphering the best parameter from an algorithm-based and the best parameter POC from an algorithm-based perspective. We conducted this study to compare three different viscoelastic POC tests: thromboelastography (TEG), rotational thromboelastometry (ROTEM), and Sonoclot analyzer to predict thrombocytopenia and hypofibrinogenemia in cyanotic cardiac surgery patients.

Material and Methods A total of 105 patients of either sex, who were scheduled to undergo elective cardiac surgery for CCHD on cardiopulmonary bypass (CPB), were enrolled after obtaining written and informed consent. Blood samples for TEG, ROTEM, Sonoclot, and standard laboratory coagulation tests were collected after induction of anesthesia (T1) and 30 minutes after protamine reversal (T2).

Results We observed significant correlations between POC parameters, platelet count, and serum fibrinogen levels. Area under the curve (AUC, 0.90) of ROTEM FIBTEM-A10 was found to be superior in detecting hypofibrinogenemia (serum fibrinogen < $200 \mathrm{mg} / \mathrm{dL}$ ). AUC of TEG $\alpha$ angle (AUC 0.79), TEG MA (AUC 0.77) and Sonoclot CR (AUC 0.73) were comparable. Sonoclot PF was found to have highest AUC (0.95) to detect thrombocytopenia (platelet count $<100,000 / \mu \mathrm{L}$ ). ROTEM FIBTEM-A10 at cutoff value $\leq 7.5 \mathrm{~mm}$ had highest sensitivity $(87.2 \%)$ and specificity $(80.3 \%)$ to detect hypofibrinogenemia. Sonoclot PF at cutoff value $\leq 0.95$ had highest sensitivity $(100 \%)$ and specificity $(83.7 \%)$ to detect thrombocytopenia. We formulated a POC algorithm based on cutoff value derived from ROC curves.

Conclusion In conclusion, although all three viscoelastic POC devices (TEG, ROTEM, and Sonoclot) can be used to detect hypofibrinogenemia and thrombocytopenia, it was reaffirmed on a larger subset of patients that ROTEM FIBTEM has highest diagnostic accuracy for hypofibrinogenemia, whereas Sonoclot PF has highest diagnostic value for thrombocytopenia in CCHD surgical patients.
DOI https://doi.org/ $10.1055 / \mathrm{s}-0039-1692118$ ISSN 2457-0206.
Copyright @2018 Official Publication of The Simulation Society (TSS), accredited by International Society of Cardiovascular Ultrasound (ISCU).
License terms

(ㅇ) (1) $\odot \circledast$ 


\section{Introduction}

Patients with cyanotic congenital heart disease (CCHD) have multifactorial hematologic abnormalities such as thrombocytopenia, platelet dysfunction, and deficiency of coagulation factors. ${ }^{1-6}$ These abnormalities are further accentuated due to hemodilution and platelet dysfunction during cardiopulmonary bypass (CPB)., ${ }^{7,8}$ This leads to increased bleeding tendency during surgery and postoperative period. Prediction of these hematologic abnormalities can identify patients at risk and optimize hemostatic therapy.

Conventional laboratory tests are not of much use during urgent situations because of prolonged turnaround time and inability to predict bleeding. ${ }^{9}$ Empirical treatment might lead to unnecessary blood product transfusion or suboptimal hemostatic management. Today, viscoelastic point-of-care (POC) methods such as thromboelastography (TEG), rotational thromboelastometry (ROTEM), and Sonoclot analyzer (Sienco Inc.) are available. ${ }^{10}$ The use of viscoelastic POC devices to guide hemostatic therapy has been demonstrated to decrease blood products requirement and improve clinical outcome. ${ }^{11-16}$

We conducted this study to evaluate diagnostic accuracy of three different viscoelastic POC tests (TEG, ROTEM, and Sonoclot analyzer) to detect thrombocytopenia and hypofibrinogenemia in cyanotic cardiac surgery patients following an algorithm (AIIMS POC algorithm).

\section{Material and Methods}

\section{Study Design and Subjects}

This prospective observational study was conducted after approval from institutional ethics committee (IECPG/394/5)..$^{17}$ Total 105 patients of either sex, who were scheduled to undergo elective cardiac surgery for CCHD on CPB, were enrolled after obtaining written and informed consent. Patients on preoperative anticoagulants and patients undergoing cardiac surgery without involving СРB were not enrolled in this study.

\section{Methodology}

Preanesthetic evaluation was done 1 day prior to surgery. Anesthetic induction consisted of intravenous (IV) ketamine (2 mg/kg)/oxygen-sevoflurane mixture, IV fentanyl ( $2-3 \mu \mathrm{g} / \mathrm{kg}$ ), and rocuronium bromide $(1 \mathrm{mg} / \mathrm{kg})$. Anesthesia was maintained with isoflurane $(0.5-1.5 \%)$ in oxygen-air mixture with intermittent doses of IV fentanyl, midazolam, and pancuronium. Monitoring of five-lead electrocardiogram, arterial blood pressure, pulse oximetry, central venous pressure, nasal temperature, and urine output was done in all patients. Patients were heparinized with $4 \mathrm{mg} / \mathrm{kg}$ unfractionated IV heparin to achieve a target-activated clotting time (ACT) of more than 480 seconds after aortic pursestring suture.

The priming of СРB circuit was done with Ringer's lactate solution $20 \mathrm{~mL} / \mathrm{kg}$, mannitol (20\%) $0.5 \mathrm{~g} / \mathrm{kg}$, sodium bicarbonate $(7.5 \%) 1 \mathrm{~mL} / \mathrm{kg}$, and $100 \mathrm{U} / \mathrm{kg}$ of unfractionated heparin. Patient hematocrit was maintained more than 30\% by addition of packed red blood corpuscles (PRBC) to the pump volume. Arterial blood gas analysis and ACT were performed intraoperatively at half hourly intervals. The CPB pump flows were maintained between 120 and $200 \mathrm{~mL} / \mathrm{kg} / \mathrm{min}$.

Anticoagulation was reversed with IV protamine $1.3 \mathrm{mg} / \mathrm{mg}$ of heparin after coming off $\mathrm{CPB}$. Hemostasis was maintained with transfusion of platelets, fresh frozen plasma, and cryoprecipitate after coming off CPB. Epsilon-aminocaproic acid (EACA) $100 \mathrm{mg} / \mathrm{kg}$ was administered after induction of anesthesia, during $\mathrm{CPB}$, and after coming off $\mathrm{CPB}$ in all patients. Patients were transferred to intensive care unit (ICU) for postoperative ventilation. The decision of blood product transfusion was taken by physician on the basis of surgical site bleeding in operating theater or chest drain output in ICU.

\section{Sample Collection}

Blood samples for TEG, ROTEM, Sonoclot, and standard laboratory coagulation tests were collected at two time points. The first blood sample $(12 \mathrm{~mL})$ was collected after induction of anesthesia (T1), and the second sample was collected 30 minutes after protamine reversal (T2). Blood samples for TEG and ROTEM analysis were drawn into $3.2 \%$ sodium citrate Vacutainer (2.7 mL; Becton, Dickinson and Company) and analyzed immediately after recalcification with $20 \mu \mathrm{L}$ of $0.2 \mathrm{M}$ calcium chloride. Blood samples for Sonoclot were taken into plain syringes and used immediately for analysis.

Blood sample for hemoglobin concentration and platelet count was collected into $4 \mathrm{~mL}$ spray-dried ethylenediaminetetraacetate (EDTA)-K2 Vacutainer and measured in a hematology analyzer (Siemens Advia 2120i hematology system; Siemens Healthcare Private Limited), whereas blood sample for coagulation tests such as prothrombin time (PT), international normalized ratio (INR), activated thromboplastin time (aPTT), and fibrinogen concentration were collected in $3.2 \%$ sodium citrate Vacutainer $(2.7 \mathrm{~mL})$ and measured with coagulation analyzer (STA Compact; Diagnostica Stago). Serum fibrinogen concentration was measured using modified Clauss method.

\section{Viscoelastic Point-of-Care Measurements}

Sonoclot signature was obtained and parameters such as Sonoclot ACT (SON ACT), clot rate (CR), and platelet function (PF) were recorded on Sonoclot analyzer. The extrinsic rotational thromboelastometry (EXTEM), intrinsic thromboelastometry (INTEM), and fibrinogen thromboelastometry (FIBTEM) were performed with rotational thromboelastometry analyzer (ROTEM, Tem System). ROTEM EXTEM parameters such as clotting time (CT), clot formation time (CFT), clot amplitude at 5 minutes (A5), clot amplitude at 10 minutes (A10), maximum clot firmness (MCF), and maximum clot elasticity (MCE) were noted. Similarly, ROTEM INTEM parameters (CT, CFT, MCF) and FIBTEM parameters (A5, A10, MCF, MCE) were also recorded. ROTEM MCE was calculated by subtracting FIBTEM MCE from EXTEM MCE. Thromboelastography (TEG) measurements such as reaction time $(R)$, kinetics $(K), \alpha$ angle, and maximum clot amplitude (MA) were recorded on dual-channel, computed TEG (Haemoscope Corp.) device.

\section{Parameters Evaluated}

Patient demographic data and perioperative parameters such as age, sex, height, body weight, type of surgical procedure, duration of $\mathrm{CPB}$, and number of blood product 
transfused (fresh frozen plasma, random donor platelets, cryoprecipitate) were collected. The baseline heart rate, arterial blood pressure, pulse oximetry, central venous pressure, echocardiography, and routine hematologic investigations were also noted. Once the patient was shifted to the ICU, data on postoperative chest drain output (CDO) hourly for 24 hours, blood product transfusion, duration of mechanical ventilation, duration of ICU stay, and duration of hospital stay were observed.

\section{Statistical Analysis}

Continuous data are presented as mean and interquartile range (IQR). The POC parameters at baseline (T1) and 30 minutes after protamine administration (T2) were compared using Wilcoxon signed ranked test. Pearson's correlation coefficient was used to assess correlation between viscoelastic POC parameters and standard laboratory tests. Receiver operating characteristic (ROC) curve analysis was used to determine cutoff value, sensitivity, and specificity of POC parameters to detect thrombocytopenia (platelet count $<100,000 / \mu \mathrm{L}$ ) and hypofibrinogenemia (serum fibrinogen concentration $<200 \mathrm{mg} / \mathrm{dL}$ ). The optimal cutoff values of POC parameters were determined using Youden's index. ${ }^{18}$ A $p<0.05$ was considered statistically significant. Statistical analysis was performed using computer software statistical package for social sciences (SPSS version 21.0, Inc.).

\section{Results}

The demographic characteristics and perioperative parameter of 105 patients included in this study are shown in - Table $\mathbf{1}$. Patients undergoing CCHD repair including tetralogy of Fallot repair $(n=77)$, Glenn procedure $(n=9)$, Fontan procedure $(n=6)$, arterial switch over $(n=7)$, and total anomalous pulmonary venous connections $(n=6)$ were included. The mean $( \pm$ SD) CPB time was $115.13( \pm 49.74)$ minutes. We observed statistically significant difference between POC parameters after anesthesia induction and 30 minutes after protamine administration indicating CPB-related hematologic changes (- Table 2).

The correlations between baseline (T1) POC parameters and standard laboratory tests are shown in - Table 3 . We observed strong correlation between serum fibrinogen and ROTEM FIBTEM-A10 ( $r: 0.779 ; p<0.001)$ and FIBTEM MCF ( $r: 0.755$; $p<0.001)$. The serum fibrinogen also showed moderate correlation with TEG $\alpha$ angle $(r: 0.481 ; p<0.001)$, TEG MA ( $r: 0.406 ; p<0.001)$, and Sonoclot CR $(r: 0.437 ; p<0.001)$, respectively. Similarly, we found moderate correlation between Sonoclot PF ( $r: 0.513 ; p<0.001)$ and platelet count. However, weak correlation was seen between platelet count and ROTEM EXTEM-A5 ( $r: 0.315 ; p<0.001)$, EXTEM-A10 ( $r$ : $0.241 ; p<0.05)$, and TEG MA $(r: 0.239 ; p<0.05)$. We observed poor correlation of platelet count with ROTEM INTEM MCF $(r$ : $0.14 ; p=0.157)$ and ROTEM-derived MCE $(r: 0.11 ; p=0.273)$.

Area under the curves (AUC) of ROC analysis was observed to evaluate the ability of ROTEM, TEG, and Sonoclot to detect thrombocytopenia and hypofibrinogenemia, whereas AUC (0.93) of ROTEM FIBTEM-A10 was found to be superior to
Table 1 Demographics and perioperative data of studied population $(n=105)^{*}$

\begin{tabular}{|c|c|}
\hline Age (y) & $6.31(0.08-34)$ \\
\hline $\operatorname{Sex}(M / F)$ & $72 / 33$ \\
\hline Height (cm) & $101.76(45-166)$ \\
\hline Weight (kg) & $17.55(2.7-55)$ \\
\hline \multicolumn{2}{|l|}{ Preoperative variables } \\
\hline $\begin{array}{l}\text { Baseline hematological parameter } \\
\text { HCT }(\%) \\
\text { Platelet count (per } \mu \mathrm{L}) \\
\text { Prothrombin time }(\mathrm{s}) \\
\text { Activated partial thromboplastin } \\
\text { time }(\mathrm{s}) \\
\text { Fibrinogen }(\mathrm{mg} / \mathrm{dL})\end{array}$ & $\begin{array}{l}53.09(29.2-80.4) \\
211,000 \\
(29,000-498,000) \\
15.3(10.7-34.5) \\
40.77(15.3-85.2) \\
242.48(88-390)\end{array}$ \\
\hline \multicolumn{2}{|l|}{ Intraoperative variables } \\
\hline $\begin{array}{l}\text { Duration of CPB (min) } \\
\text { Cross clamp time (min) }\end{array}$ & $\begin{array}{l}115.13(32-292) \\
66.4(23-192)\end{array}$ \\
\hline $\begin{array}{l}\text { Operation type } \\
\text { Intracardiac repair (TOF) } \\
\text { Glenn } \\
\text { Fontan } \\
\text { TAPVC repair } \\
\text { ASO }\end{array}$ & $\begin{array}{l}- \\
77 \\
9 \\
6 \\
6 \\
7\end{array}$ \\
\hline $\begin{array}{l}\text { Transfusion (Intra- and postoper- } \\
\text { ative } 24 \text { h) } \\
\text { PRBC (units) } \\
\text { FFP (units) } \\
\text { Platelets (units) } \\
\text { Cryoprecipitate (units) }\end{array}$ & $\begin{array}{l}1.09(0-7) \\
1.24(0-8) \\
2.12(0-11) \\
0.37(0-8)\end{array}$ \\
\hline \multicolumn{2}{|l|}{ Postoperative variables } \\
\hline $\begin{array}{l}\text { Total CDO in ICU } \\
6 \mathrm{~h}(\mathrm{~mL} / \mathrm{kg}) \\
24 \mathrm{~h}(\mathrm{~mL} / \mathrm{kg})\end{array}$ & $\begin{array}{l}4.35(0-20.40) \\
11.79(0-54.80)\end{array}$ \\
\hline Ventilation duration (h) & $44.31(2-720)$ \\
\hline ICU duration (days) & $4.55(1.5-30)$ \\
\hline Hospital duration (days) & $10.82(5-40)$ \\
\hline
\end{tabular}

Abbreviations: ASO, arterial switchover; CDO, chest drain output; FFP, fresh frozen plasma; $\mathrm{HCT}$, hematocrit; ICU, intensive care unit; PRBC, packed red blood cells; TAPVC, total anomalous pulmonary venous connections; TOF, tetralogy of Fallot.

* Data are presented as mean (interquartile range) where appropriate.

other POC parameters for assessing hypofibrinogenemia (serum fibrinogen $<200 \mathrm{mg} / \mathrm{dL}$ ) ( - Fig. 1). AUC (0.79) of TEG $\alpha$ angle, AUC ( 0.77$)$ of TEG MA, and AUC (0.73) of Sonoclot CR were found to be comparable to each other.

Whereas Sonoclot PF was found to have highest AUC (0.95) to predict thrombocytopenia (platelet count $<100,000 / \mu \mathrm{L}$ ), AUC (0.70) of TEG MA was comparable to AUC of EXTEM A10 (AUC 0.70) (-Fig. 2), the ROTEM-derived MCE parameter was found to have lowest AUC to predict thrombocytopenia (platelet count $<100,000 / \mu \mathrm{L}$ ).

The sensitivity, specificity, positive predictive value (PPV), and negative predictive value (NPV) of baseline POC (ROTEM, TEG, Sonoclot) parameters to predict thrombocytopenia and hypofibrinogenemia are shown in - Table 4 . The cutoff values of baseline POC parameters to predict thrombocytopenia and hypofibrinogenemia were calculated from ROC curves using 
Table 2 ROTEM, TEG, and Sonoclot parameters at baseline (T1) and 30 minutes after protamine administration $(T 2)(n=105)^{* *}$

\begin{tabular}{|c|c|c|}
\hline POC parameters & T1 & T2 \\
\hline $\begin{array}{l}\text { ROTEM } \\
\text { EXTEM CT }(\mathrm{s})^{*} \\
\text { EXTEM CFT }(\mathrm{s})^{*} \\
\text { EXTEM-A5 }(\mathrm{mm})^{*} \\
\text { EXTEM-A10 }(\mathrm{mm})^{*} \\
\text { EXTEM MCF }(\mathrm{mm})^{*} \\
\text { MCE }(\mathrm{mm})^{*} \\
\text { INTEM CT(s) } \\
\text { INTEM CFT }(\mathrm{s})^{*} \\
\text { INTEM MCF }(\mathrm{mm})^{*} \\
\text { FIBTEM-A5 }(\mathrm{mm})^{*} \\
\text { FIBTEM-A10 }(\mathrm{mm})^{*} \\
\text { FIBTEM MCF }(\mathrm{mm})^{*}\end{array}$ & $\begin{array}{l}140.26 \\
(48-1108) \\
250.31 \\
(48-1619) \\
28.35(5-52) \\
40.0(6-62) \\
47.41(7-69) \\
90.79(4-196) \\
271.25 \\
(103-1060) \\
227.41 \\
(53-1758) \\
45.68(10-71) \\
6.98(1-18) \\
8.27(2-20) \\
9.22(2-23)\end{array}$ & $\begin{array}{l}159.83(64-670) \\
280.82(60-998) \\
23.29(5-49) \\
33.87(6-59) \\
40.51(7-68) \\
65.94(6-164) \\
284.46 \\
(122-660) \\
273.68(72-953) \\
39.49(14-101) \\
5.95(1-19) \\
7.01(1-20) \\
7.69(2-22)\end{array}$ \\
\hline $\begin{array}{l}\text { Sonoclot } \\
\text { SON ACT(s)* } \\
\text { SON CR }(U / \mathrm{min})^{*} \\
\text { SON PF }\end{array}$ & $\begin{array}{l}130.66(52-565) \\
15.22(0.5-38) \\
1.45(0-4)\end{array}$ & $\begin{array}{l}142.75(60-291) \\
13.64(1.1-44) \\
1.11(0.1-2.7)\end{array}$ \\
\hline $\begin{array}{l}\text { TEG } \\
\text { TEG R }(\min )^{*} \\
\text { TEG K }(\min )^{*} \\
\text { TEG MA }(\mathrm{mm})^{*}\end{array}$ & $\begin{array}{l}8.23(35-26.2) \\
4.12(1.1-19) \\
48.74(12.4-67.5)\end{array}$ & $\begin{array}{l}8.75(2.8-20.9) \\
4.64(1.1-12.9) \\
45.38(18.2-83)\end{array}$ \\
\hline
\end{tabular}

Abbreviations: A5, clot amplitude at 5 minutes; A10, clot amplitude at 10 minutes; $A C T$, activated clotting time; CFT, clot formation time; CR, clot rate; $\mathrm{CT}$, clot time; EXTEM, extrinsically activated thromboelastometry; FIBTEM, fibrin-specific thromboelastometry; INTEM, intrinsically activated thromboelastometry; K, clot kinetics; MA, maximum amplitude; MCE, maximum clot elasticity; MCF, maximum clot firmness; PF, platelet function; R, reaction time; ROTEM, rotational thromboelastometry; TEG, thromboelastography. * Statistically significant $(p<0.0001)$.

** Data are presented as mean (interquartile range) where appropriate.
Youden's index..$^{18}$ The ROTEM FIBTEM-A10 at threshold value $\leq$ $7.5 \mathrm{~mm}$ had highest sensitivity (87.2\%) and specificity (80.3\%) to predict hypofibrinogenemia. The Sonoclot PF at cutoff value $\leq 0.95$ had highest sensitivity $(100 \%)$ and specificity (83.7\%) to predict thrombocytopenia. All POC parameters had high NPV and low PPV.

We formulated a POC algorithm based on cutoff value derived from ROC curves ( - Fig. 3). The most appropriate cutoff value was determined using Youden's index. ${ }^{17}$ We selected that value of the ROC curve as a cutoff value, where Youden's index (sensitivity + specificity-1) was maximal. The threshold value of EXTEM CT, INTEM CT, and TEG R is taken as $20 \%$ above the upper normal reference limit. ${ }^{19,20}$

\section{Discussion}

Viscoelastic POC instruments provide rapid assessment of coagulation cascade from beginning of clot formation to clot lysis. The use of POC-guided algorithm has been shown to decrease the requirement of blood product transfusion. ${ }^{14-16}$ These POC devices have shown good correlation with standard laboratory tests. ${ }^{21-23}$

Ogawa et al conducted a prospective observational study to compare ROTEM-derived variables with standard laboratory tests in 26 patients, who were scheduled to undergo elective cardiac surgery. The author observed strong correlations between FIBTEM-A10 and fibrinogen level $(r=0.87$; $p<0.001)$ and between EXTEM/INTEM-A10 variables and platelet count $(r=0.72$ and 0.67 , respectively; $p<0.001$ ). ROC analysis demonstrated that EXTEM-A10 and INTEM-A10 are predictive of thrombocytopenia below $80 \times 10^{9} / \mathrm{L}$ (AUC, 0.83 and 0.82 , respectively), and FIBTEM-A10 was highly predictive of fibrinogen level below $200 \mathrm{mg} / \mathrm{dL}$ (AUC, 0.96). ${ }^{21}$

Table 3 Correlation between baseline point-of-care (ROTEM, TEG, Sonoclot) parameters and standard laboratory tests

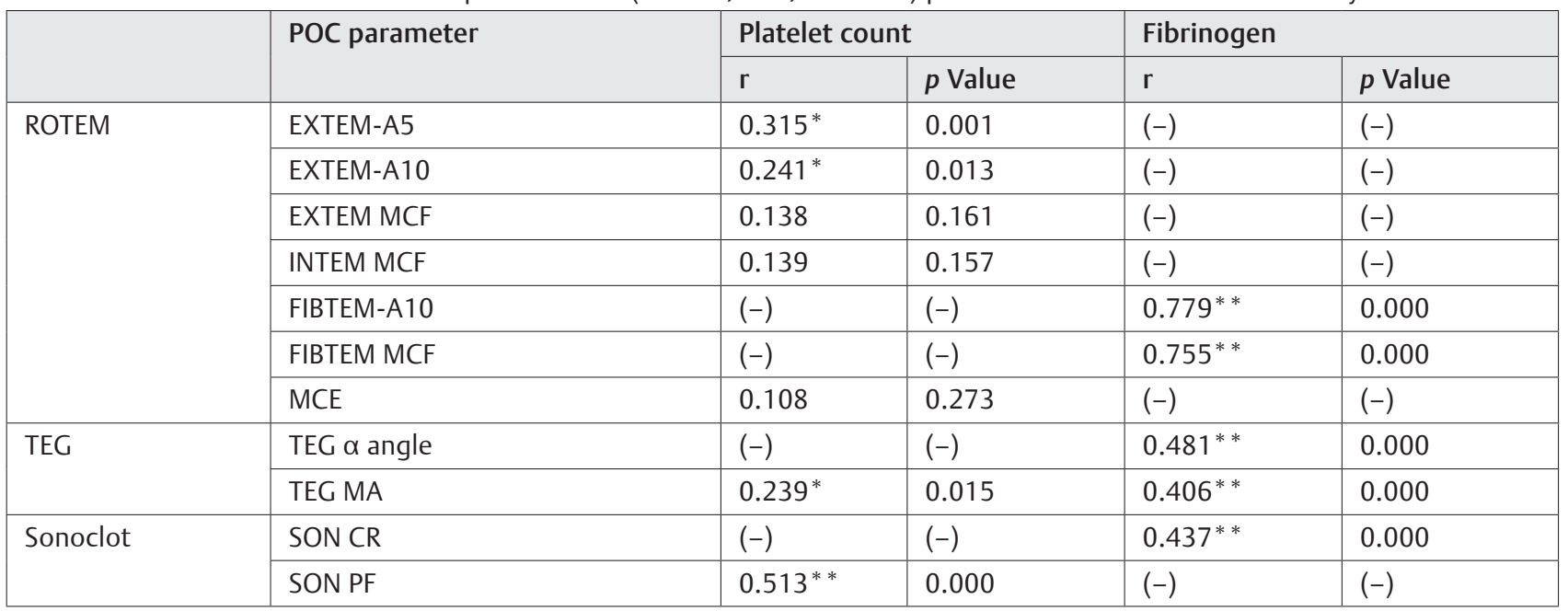

Abbreviations: (-), data not calculated; A5, clot amplitude at 5 minutes; A10, clot amplitude at 10 minutes; ACT, activated clotting time; $r$, correlation coefficient; CR, clot rate; EXTEM, extrinsically activated thromboelastometry; FIBTEM, fibrin-specific thromboelastometry; INTEM, intrinsically activated thromboelastometry; K, clot kinetics; MA, maximum amplitude; MCE, maximum clot elasticity; MCF, maximum clot firmness; PF, platelet function; POC, point of care; R, reaction time; ROTEM, rotational thromboelastometry; TEG, thromboelastography.

${ }^{*} p<0.05$.

${ }^{* *} p<0.001$. 


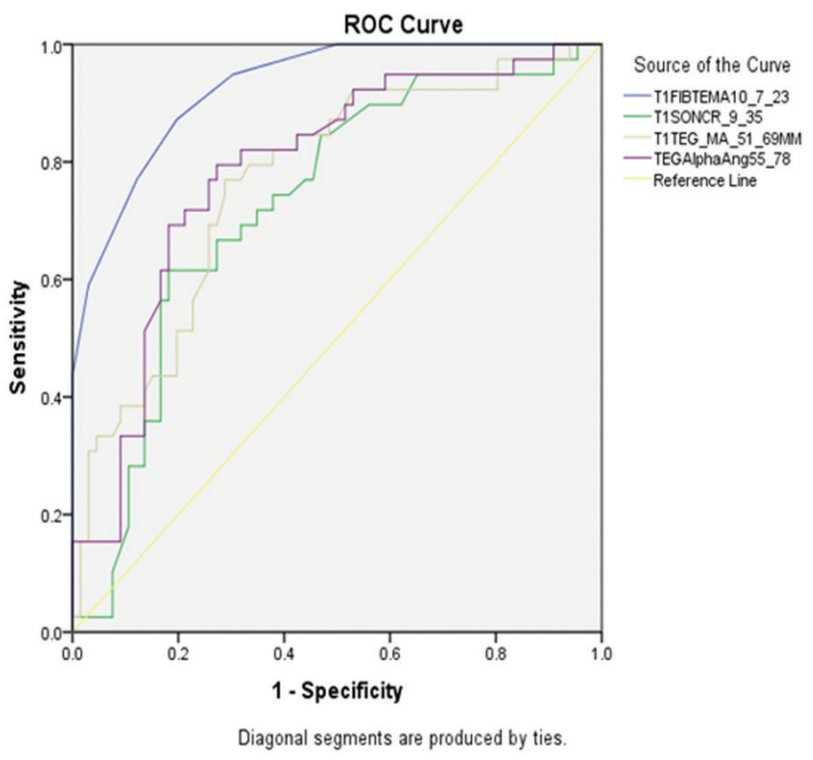

Fig. 1 Receiver operating characteristic curves of the ability of FIBTEM-A10, TEG $\alpha$ angle, TEG MA, and Sonoclot CR to predict hypofibrinogenemia (serum fibrinogen $<200 \mathrm{mg} / \mathrm{dL}$ ). FIBTEM-A10 (AUC 0.93) is superior whereas TEG $\alpha$ angle (AUC 0.78), TEG MA (AUC 0.77) and Sonoclot CR (AUC 0.73) are comparable in predicting hypofibrinogenemia. A10, amplitude at 10 minutes; FIBTEM, fibrin-specific thromboelastometry; CR, clot rate; MA, maximum amplitude; TEG, thromboelastography.

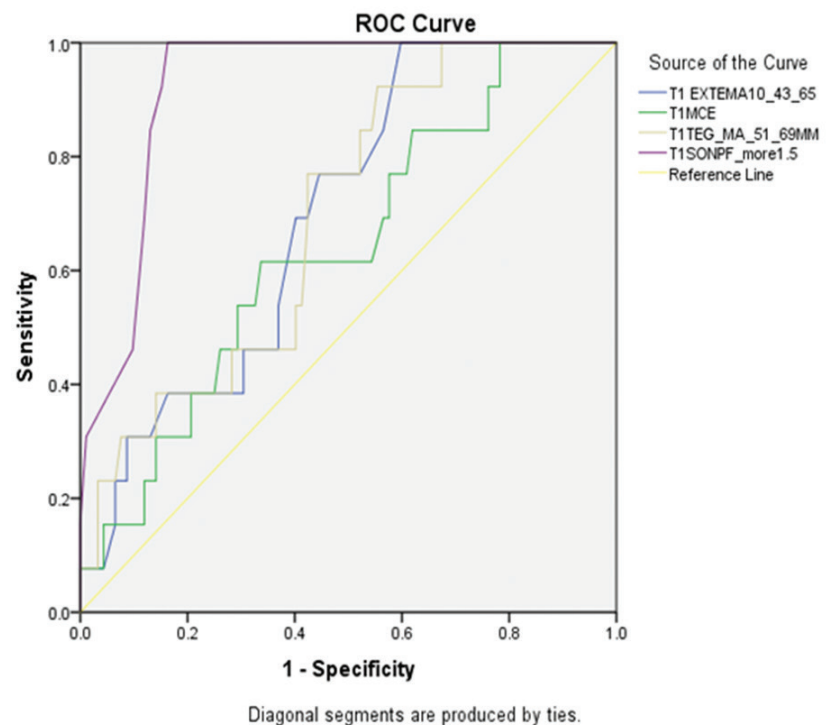

Fig. 2 Receiver operating characteristic curves of the ability of EXTEMA10, ROTEM MCE, TEG MA, and Sonoclot PF to predict thrombocytopenia (platelet count $<100,000 / \mu \mathrm{L}$ ). Sonoclot PF (AUC 0.92) is superior to EXTEM-A10 (AUC 0.70), ROTEM MCE (AUC 0.64), and TEG MA (AUC 0.70) in predicting thrombocytopenia. A10, amplitude at 10 minutes; EXTEM, extrinsically activated thromboelastometry; MA, maximum amplitude; MCE, maximum clot elasticity; SONPF, Sonoclot platelet function.

Saxena et al also assessed the correlations between Sonoclot variables and conventional coagulation tests. Clinical and laboratory data from 50 patients of four subgroups of liver disease, including decompensated cirrhosis, chronic hepatitis, cirrhosis with hepatocellular carcinoma (HCC), and acute-on-chronic liver failure, were analyzed. The authors observed significant positive correlation between Sonoclot CR and serum fibrinogen $(r=0.39$; $p<0.004)$. The Sonoclot PF also showed strong correlation with platelet count $(r=0.62 ; p<0.0001) .^{22}$
Espinosa et al conducted a prospective observational study in adult patients undergoing elective cardiac surgery with CPB. The author investigated possible correlations between TEG, ROTEM, Sonoclot variables, and routine hematologic coagulation tests. They observed significant correlations between FIBTEM MCF $(r=0.79 ; p<0.0005)$, TEG MA $(r=0.76$; $p<0.0005)$, and plasma fibrinogen levels. Similarly platelet count was demonstrated to correlate with TEG MA $(r=0.44$, $p=0.009)$ and ROTEM EXTEM MCF $(r=0.53 ; p<0.001)$. The authors did not study the correlation of Sonoclot PF with platelet count. ${ }^{23}$

In our study also, we observed strong correlation between ROTEM FIBTEM-A10 and serum fibrinogen concentration $(r=0.78 ; p<0.001)$ and moderate correlation between Sonoclot PF and platelet count $(r=0.51 ; p<0.001)$. The TEG parameters such as TEG MA and TEG $\alpha$ angle showed statistically significant correlation with serum fibrinogen $(r=0.41 ; p<0.001$ and $r=0.48 ; p<0.001)$. Platelet count also showed statistically significant correlation with EXTEM A5/A10 $(r=0.31 ; p=0.001 / r=0.24 ; p=0.013)$ and TEG MA $(r=0.24 ; p=0.015)$.

Olde et al investigated the ability of ROTEM to predict thrombocytopenia and hypofibrinogenemia in cardiac surgery patients using A5. The authors observed that FIBTEM-A5 can predict hypofibrinogenemia (fibrinogen $<200 \mathrm{mg} / \mathrm{dL}$ ) with $100 \%$ sensitivity, $42 \%$ specificity, and ROC AUC 0.95 . Similarly, EXTEM-A5 was found to predict thrombocytopenia (platelet count $<1,00,000 / \mu \mathrm{L}$ ) with $100 \%$ sensitivity, $47 \%$ specificity, and ROC AUC 0.87.24

In our study, we compared the ability of ROTEM, TEG, and Sonoclot analyzer to predict thrombocytopenia (platelet count $<100,000 / \mu \mathrm{L}$ ) and hypofibrinogenemia (serum fibrinogen $<200 \mathrm{mg} / \mathrm{dL}$ ) in patients undergoing repair of CCHD under CPB. We observed that while FIBTEM-A10 has strong ability to predict hypofibrinogenemia (ROC AUC, 0.93), Sonoclot PF has moderate ability to predict thrombocytopenia (ROC AUC, 0.92). In contrast, TEG $\alpha$ angle, TEG MA, and Sonoclot CR have moderate ability to predict hypofibrinogenemia (AUC; 0.79 , 0.77 , and 0.73 , respectively). The ROTEM EXTEM-A10, MCE, and TEG MA were observed to have moderate ability to predict thrombocytopenia (AUC; 0.70, 0.64, and 0.69, respectively). The cutoff values of POC parameters to predict thrombocytopenia and hypofibrinogenemia were determined using ROC curves and Youden's index. ${ }^{18}$ We noted that FIBTEM-A10 at threshold value $\leq 7.5 \mathrm{~mm}$ has $87.2 \%$ sensitivity and $80.3 \%$ specificity to predict hypofibrinogenemia, whereas Sonoclot PF at threshold value $\leq 0.95$ has $100 \%$ sensitivity and $83.7 \%$ specificity to predict thrombocytopenia. The difference in threshold value, sensitivity, specificity, and AUC noted in our study from previous study could be due to difference in studied population (cyanotic vs. acyanotic cardiac surgery patients). We propose a POC-guided algorithm using cutoff values of ROTEM, TEG, and Sonoclot parameters to guide hemostatic therapy in cyanotic cardiac surgery patients (-Fig. 3). However, the efficacy of this algorithm should be studied in a randomized controlled trial.

Our study has few limitations. The platelet aggregometry POC tests were not performed in this study. This 
Table 4 Diagnostic accuracy of baseline ROTEM, TEG, and SON parameters to detect thrombocytopenia (platelet count < 100,000/ $\mu \mathrm{L}$ ) and hypofibrinogenemia (serum fibrinogen $<200 \mathrm{mg} / \mathrm{dL}$ )

\begin{tabular}{|c|c|c|c|c|c|c|c|}
\hline & $\begin{array}{l}\text { POC parameter } \\
\text { (threshold value) }\end{array}$ & $\begin{array}{l}\text { ROC } \\
\text { AUC }\end{array}$ & Sensitivity & Specificity & PPV & NPV & $\begin{array}{l}95 \% \\
\mathrm{Cl}\end{array}$ \\
\hline \multirow[t]{4}{*}{$\begin{array}{l}\text { Fibrinogen } \\
(<200 \mathrm{mg} / \mathrm{dL})\end{array}$} & $\begin{array}{l}\text { FIBTEM-A10 } \\
(\leq 7.5 \mathrm{~mm})\end{array}$ & 0.93 & 87.2 & 80.3 & 72.3 & 91.4 & $0.88-0.97$ \\
\hline & $\begin{array}{l}\text { TEG } \alpha \text { angle } \\
\text { ( } \leq 48 \text { degree) }\end{array}$ & 0.79 & 76.9 & 72.7 & 62.5 & 84.2 & $0.69-0.88$ \\
\hline & $\begin{array}{l}\text { TEG MA } \\
(\leq 50.3 \mathrm{~mm})\end{array}$ & 0.77 & 76.9 & 71.2 & 61.2 & 83.9 & $0.67-0.86$ \\
\hline & $\begin{array}{l}\text { SON CR } \\
(\leq 13 \mathrm{U} / \mathrm{min})\end{array}$ & 0.73 & 71.8 & 65.2 & 54.9 & 79.6 & $0.63-0.83$ \\
\hline \multirow[t]{4}{*}{$\begin{array}{l}\text { Platelet } \\
(<100,000 / \mu \mathrm{L})\end{array}$} & $\begin{array}{l}\text { EXTEM-A10 } \\
(\leq 42.5 \mathrm{~mm})\end{array}$ & 0.70 & 76.9 & 55.4 & 19.6 & 94.4 & $0.57-0.83$ \\
\hline & $\begin{array}{l}\text { MCE } \\
(\leq 102 \mathrm{~mm})\end{array}$ & 0.64 & 76.9 & 42.4 & 15.9 & 92.8 & $0.49-0.79$ \\
\hline & $\begin{array}{l}\text { TEG MA } \\
(\leq 54.9 \mathrm{~mm})\end{array}$ & 0.70 & 92.3 & 44.6 & 18.3 & 95.5 & $0.56-0.83$ \\
\hline & SON PF $(\leq 0.95)$ & 0.92 & 100 & 83.7 & 46.42 & 100 & $0.87-0.97$ \\
\hline
\end{tabular}

Abbreviations: A10, clot amplitude at 10 minutes; AUC, area under the curve; $\mathrm{Cl}$, confidence interval; CR, clot rate; EXTEM, extrinsically activated thromboelastometry; FIBTEM, fibrin-specific thromboelastometry; MA, maximum amplitude; MCE, maximum clot elasticity; NPV, negative predictive value; PF, platelet function; PPV, positive predictive value; ROC, receiver operating characteristic; ROTEM, rotational thromboelastometry; SON, Sonoclot; TEG, thromboelastography.

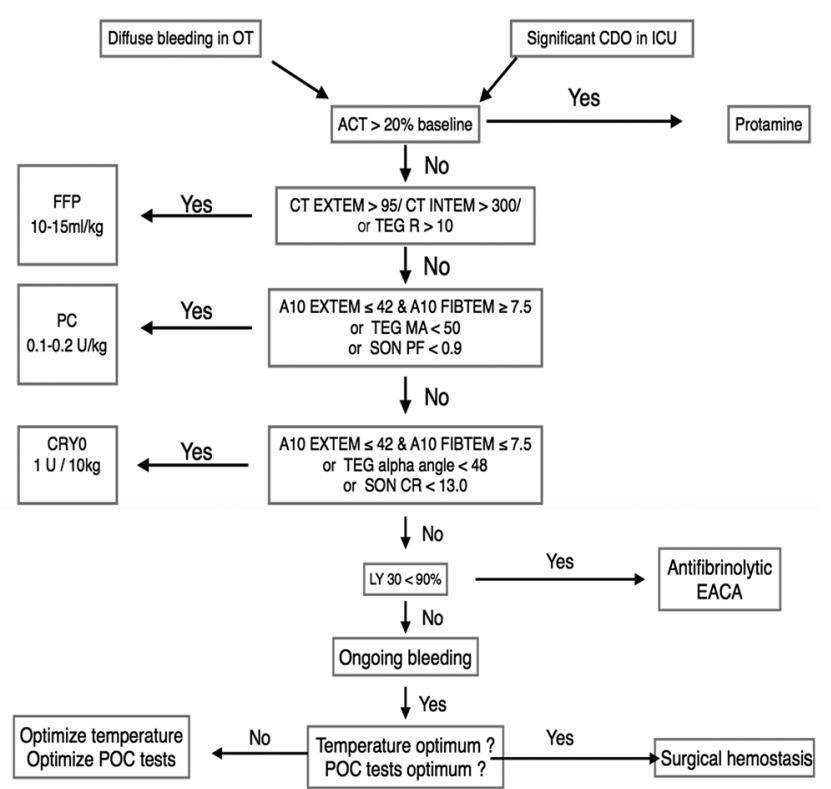

Fig. 3 Point-of-care (POC) algorithm using three viscoelastic POC devices; (ROTEM, TEG, and Sonoclot analyzer) to guide hemostatic therapy in cyanotic congenital heart disease patients. A10, amplitude at 10 minutes; $\mathrm{ACT}$, activated clotting time; $\mathrm{CR}$, clot rate; $\mathrm{CT}$, clotting time; CDO, chest drain output; CRY0, cryoprecipitate; EACA, epsilon amino caproic acid; EXTEM, extrinsically activated thromboelastometry; FFP, fresh frozen plasma; FIBTEM, fibrin-specific thromboelastometry; ICU, intensive care unit; INTEM, intrinsically activated thromboelastometry; LY30, clot lysis after 30 minutes; MA, maximum amplitude; OT, operation theater; PC, platelet concentrate; PF, platelet function; R, reaction time; SON, Sonoclot; TEG, thromboelastography.

study was limited to cyanotic cardiac surgery patients; acyanotic cardiac patients were not included. Therefore, this study results might not be valid in acyanotic cardiac surgery patients. This study was planned to evaluate diagnostic accuracy of these viscoelastic POC tests to detect thrombocytopenia and hypofibrinogenemia. The efficacy of POC devices to guide hemostatic therapy and its effect on surgical outcome was not performed in this study. A randomized control study would be required to evaluate efficacy of these three POC tests on surgical outcome in CCHD repair.

\section{Conclusion}

In conclusion, although all three viscoelastic POC devices (TEG, ROTEM, and Sonoclot) can be used to detect hypofibrinogenemia and thrombocytopenia: ROTEM FIBTEM has highest diagnostic accuracy for hypofibrinogenemia, whereas Sonoclot PF has highest diagnostic value for thrombocytopenia in patients undergoing surgery for CCHD. In future, randomized controlled studies are required to evaluate efficacy of these tests on surgical outcome in CCHD patients. Use of these POC tests helps choose the right blood component therapy to reduce bleeding in these cyanotic children undergoing corrective surgery.

\section{Conflicts of Interest}

None.

\section{Acknowledgment}

This investigation is being funded by research grant under the JCCC-TSS banner. The research aid was provided for equipment and reagents by Vijyoti Management, New Delhi. 


\section{References}

1 Kumor-Kurek M, Siudalska H. Hematologic disorders in patients with cyanotic congenital heart disease. Pol Merkuriusz Lek 1999;7(38):82-4

2 Chamanian S, Maleki M, Rohani A, Amini M. Hematologic abnormalities in cyanotic congenital heart disease patients. J Cardiothorac Med 2015;3(1):270-272

3 Ghasemi A, Horri M, Salahshour Y. Coagulation abnormalities in pediatric patients with congenital heart disease: a literature review. Int J Pediatr 2014;2(2.2):141-143

4 Horigome H, Hiramatsu Y, Shigeta O, Nagasawa T, Matsui A. Overproduction of platelet microparticles in cyanotic congenital heart disease with polycythemia. J Am Coll Cardiol 2002;39(6):1072-1077

5 Mauer HM, McCue CM, Caul J, Still WJ. Impairment in platelet aggregation in congenital heart disease. Blood 1972;40(2):207-216

6 Ismail EA, Youssef OI. Platelet-derived microparticles and platelet function profile in children with congenital heart disease. Clin Appl Thromb Hemost 2013;19(4):424-432

7 Bolliger D, Szlam F, Levy JH, Molinaro RJ, Tanaka KA. Haemodilution-induced profibrinolytic state is mitigated by fresh-frozen plasma: implications for early haemostatic intervention in massive haemorrhage. Br J Anaesth 2010;104(3): 318-325

8 Despotis GJ, Gravlee G, Filos K, Levy J. Anticoagulation monitoring during cardiac surgery: a review of current and emerging techniques. Anesthesiology 1999;91(4):1122-1151

9 Segal JB, Dzik WH; Transfusion Medicine/Hemostasis Clinical Trials Network. Paucity of studies to support that abnormal coagulation test results predict bleeding in the setting of invasive procedures: an evidence-based review. Transfusion 2005;45(9):1413-1425

10 Enriquez LJ, Shore-Lesserson L. Point-of-care coagulation testing and transfusion algorithms. Br J Anaesth 2009;103 (Suppl 1):i14-i22

11 Spalding GJ, Hartrumpf M, Sierig T, Oesberg N, Kirschke CG, Albes JM. Cost reduction of perioperative coagulation management in cardiac surgery: value of "bedside" thrombelastography (ROTEM) Eur J Cardiothorac Surg 2007;31(6):1052-1057

12 Spiess BD, Gillies BSA, Chandler W, Verrier E. Changes in transfusion therapy and reexploration rate after institution of a blood management program in cardiac surgical patients. J Cardiothorac Vasc Anesth 1995;9(2):168-173
13 Ronald A, Dunning J. Can the use of thromboelastography predict and decrease bleeding and blood and blood product requirements in adult patients undergoing cardiac surgery? Interact Cardiovasc Thorac Surg 2005;4(5):456-463

14 Weber CF, Görlinger K, Meininger D, et al. Point-of-care testing: a prospective, randomized clinical trial of efficacy in coagulopathic cardiac surgery patients. Anesthesiology 2012;117(3):531-547

15 Deppe AC, Weber C, Zimmermann J, et al. Point-of-care thromboelastography/thromboelastometry-based coagulation management in cardiac surgery: a meta-analysis of 8332 patients.

16 Ak K, Isbir CS, Tetik S, et al. Thromboelastography-based transfusion algorithm reduces blood product use after elective CABG: a prospective randomized study. J Card Surg 2009;24(4):404-410

17 Bhardwaj V, Malhotra P, Hasija S, Chowdury UK, Pangasa N. Coagulopathies in cyanotic cardiac patients: an analysis with three point-of-care testing devices (thromboelastography, rotational thromboelastometry, and Sonoclot analyzer) Ann Card Anaesth 2017;20(2):212-218

18 Habibzadeh F, Habibzadeh P, Yadollahie M. On determining the most appropriate test cut-off value: the case of tests with continuous results. Biochem Med (Zagreb) 2016;26(3):297-307

19 Lang T, Bauters A, Braun SL, et al. Multi-centre investigation on reference ranges for ROTEM thromboelastometry. Blood Coagul Fibrinolysis 2005;16(4):301-310

20 Sankarankutty A, Nascimento B, Teodoro da Luz L, Rizoli S. TEG® and ROTEM ${ }^{\circledR}$ in trauma: similar test but different results? World J Emerg Surg 2012;7(Suppl 1):S3

21 Ogawa S, Szlam F, Chen EP, et al. A comparative evaluation of rotation thromboelastometry and standard coagulation tests in hemodilution-induced coagulation changes after cardiac surgery. Transfusion 2012;52(1):14-22

22 Saxena P, Bihari C, Rastogi A, Agarwal S, Anand L, Sarin SK. Sonoclot signature analysis in patients with liver disease and its correlation with conventional coagulation studies. Adv Hematol 2013;2013:237351

23 Espinosa A, Stenseth R, Videm V, Pleym H. Comparison of three point-of-care testing devices to detect hemostatic changes in adult elective cardiac surgery: a prospective observational study. BMC Anesthesiol 2014;14:80

24 Olde ERH, Kuiper GJ, Wetzels RJ, et al. Rapid and correct prediction of thrombocytopenia and hypofibrinogenemia with rotational thromboelastometry in cardiac surgery. J Cardiothorac Vasc Anesth 2014;28(2):210-216 